Journal of Mathematical Physics, Analysis, Geometry

2016, vol. 12 , No. 4, pp. 338-358

\title{
On the Spectrum of Rotating Viscous Relaxing Fluid
}

\author{
D. Zakora \\ Voronezh State University \\ 1 University Sq., Voronezh 394006, Russia \\ E-mail: dmitry_@crimea.edu, dmitry.zkr@gmail.com \\ Received October 20, 2015, revised May 11, 2016
}

In the present work, a problem on the spectrum of a viscous relaxing fluid completely filling a rotating bounded domain is formulated. The essential spectrum of the problem is obtained and the statements on the localization and a spectrum asymptotics are proven.

Key words: viscous fluid, compressible fluid, essential spectrum, complementing condition, spectrum asymptotics.

Mathematics Subject Classification 2010: 45K05, 58C40, 76R99.

\section{Introduction}

In the present paper the spectrum of a rotating viscous relaxing fluid is studied. The model of the relaxing fluid is a generalization of the barotropic fluid and is described by a system of first-order integro-differential equations. In $[1,2]$, the statements on essential and discrete spectrum of a viscous barotropic fluid are proven. In particular, it was proven that the essential spectrum of a viscous compressible fluid consists of two points.

We prove that the essential spectrum of a rotating viscous relaxing fluid consists of the finite number of segments situated on the positive real semiaxis. In the case where the system is not rotating and is in weightlessness the essential spectrum consists of the finite number of positive real numbers.

The spectrum of the problem is situated in the open right half-plane and is localized near the real axis. In the case where the system is not rotating, there can be only the finite number of nonreal eigenvalues. These nonreal eigenvalues are situated symmetrically with respect to the real axis. The rest of the spectrum

This work was supported by the grant of the Russian Foundation for Basic Research (project no. 14-21-00066), Voronezh State University.

(C) D. Zakora, 2016 
is localized on the real axis. The nonreal eigenvalues are absent if the viscosity of the fluid is sufficiently large.

Let us notice that the spectral analysis of integro-differential equations arises naturally in various problems of mathematical physics (see $[3,4]$ and references therein).

\section{Small Motions of a Rotating Viscous Relaxing Fluid}

In this section we introduce the initial-boundary value problem which describes our system. The theorem on the strong unique solvability of the initialboundary value problem is proven (Theorem 1).

\subsection{Statement of the problem}

Consider a container that uniformly rotates around the axis parallel to the gravity force and is completely filled by a viscous inhomogeneous fluid. The fluid is said to occupy a bounded region $\Omega \subset \mathbb{R}^{3}$. Let $\vec{n}$ be a unit vector perpendicular to the boundary $S:=\partial \Omega$ and directed out of the region $\Omega$. We introduce a system of coordinates $\mathrm{Ox}_{1} x_{2} x_{3}$ which is toughly connected with the container so that the $O x_{3}$ axis coincides with the rotating axis and is directed opposite the gravity force, and the origin of coordinates is in the region $\Omega$. In this case, the uniform velocity of rotation of the container takes the form $\vec{\omega}_{0}:=\omega_{0} \vec{e}_{3}$, where $\vec{e}_{3}$ is the unite vector along the rotating axis $O x_{3}$, and $\omega_{0}>0$ for definiteness. The external stationary field of forces $\vec{F}_{0}$ is considered to be a gravitational field acting along the rotating axis, i.e., $\vec{F}_{0}=-g \vec{e}_{3}, g>0$.

The problem on small motions of a rotating viscous relaxing fluid is described by the following initial-boundary value problem (see [5]):

$$
\begin{gathered}
\frac{\partial \vec{u}(t, x)}{\partial t}-2 \omega_{0}\left(\vec{u}(t, x) \times \vec{e}_{3}\right)-\rho_{0}^{-1}(z)\left(\mu \Delta \vec{u}(t, x)+\left(\eta+3^{-1} \mu\right) \nabla \operatorname{div} \vec{u}(t, x)\right) \\
+\nabla\left(a_{\infty} \rho_{0}^{-1 / 2}(z) \rho(t, x)\right)-\sum_{l=1}^{m} \int_{0}^{t} e^{-b_{l}(t-s)} \nabla\left(a_{\infty} \rho_{0}^{1 / 2}(z) k_{l}(x) \rho(s, x)\right) d s=\vec{f}(t, x), \\
\frac{\partial \rho(t, x)}{\partial t}+a_{\infty} \rho_{0}^{-1 / 2}(z) \operatorname{div}\left(\rho_{0}(z) \vec{u}(t, x)\right)=0(\text { in } \Omega), \quad \vec{u}(t, x)=\overrightarrow{0}(\text { on } S:=\partial \Omega), \\
\vec{u}(0, x)=\vec{u}^{0}(x), \quad \rho(0, x)=\rho^{0}(x),
\end{gathered}
$$

where $z:=2^{-1} \omega_{0}^{2}\left(x_{1}^{2}+x_{2}^{2}\right)-g x_{3}, \rho_{0}(z)$ is the given stationary density of the fluid (this function is smooth and satisfies the conditions $0<\alpha_{1} \leqslant \rho_{0}(z) \leqslant \alpha_{2}$ ), $a_{\infty}$ is the given sound velocity, $\vec{f}(t, x)$ is a weak field of external forces imposed on the gravitational field, $\vec{u}(t, x)$ is the field of the velocities in the fluid, $a_{\infty}^{-1} \rho_{0}^{1 / 2}(z) \rho(t, x)$ is the dynamic density of the fluid, $\mu$ and $\eta$ are the dynamic and the second viscosity of the fluid. The numbers $b_{l}^{-1}$ are used as the times of relaxation in 
the system $\left(0<b_{l}<b_{l+1}(l=\overline{1, m-1})\right)$, and $k_{l}(x)>0$ are some continuously differentiable structural functions. In the case where the system is not rotating and is in weightlessness $\left(\omega_{0}=0, g=0\right)$, the functions $k_{l}(x)$ should be considered as some constants. In this case, the function $\rho_{0}(z)$ should be also considered as a constant.

\subsection{Operator formulation of the problem}

To pass to the operator formulation of problem (1), we introduce basic spaces and a number of operators (see [5]).

Let us introduce a vector Hilbert space $\vec{L}_{2}\left(\Omega, \rho_{0}\right)$ with the scalar product and the norm

$$
(\vec{u}, \vec{v})_{\vec{L}_{2}\left(\Omega, \rho_{0}\right)}:=\int_{\Omega} \rho_{0}(z) \vec{u}(x) \cdot \overline{\vec{v}(x)} d \Omega, \quad\|\vec{u}\|_{\vec{L}_{2}\left(\Omega, \rho_{0}\right)}^{2}=\int_{\Omega} \rho_{0}(z)|\vec{u}(x)|^{2} d \Omega .
$$

We introduce a scalar Hilbert space $L_{2}(\Omega)$ of the square summable functions in the region $\Omega$, and also its subspace $L_{2, \rho_{0}}(\Omega):=\left\{f \in L_{2}(\Omega) \mid\left(f, \rho_{0}^{1 / 2}\right)_{L_{2}(\Omega)}=0\right\}$. By the formula

$$
\Pi f:=f-\left(f, \rho_{0}^{1 / 2}\right)_{L_{2}(\Omega)}\left\|\rho_{0}^{1 / 2}\right\|_{L_{2}(\Omega)}^{-2} \rho_{0}^{1 / 2}(z),
$$

we introduce an orthogonal projector of the space $L_{2}(\Omega)$ onto the subspace $L_{2, \rho_{0}}(\Omega)$.

Lemma 1. The following statements hold.

1. Let us define an operator $S \vec{u}:=i\left(\vec{u} \times \vec{e}_{3}\right), \mathcal{D}(S):=\vec{L}_{2}\left(\Omega, \rho_{0}\right)$. The operator $S$ is selfadjoint and bounded in $\vec{L}_{2}\left(\Omega, \rho_{0}\right): S=S^{*}, S \in \mathcal{L}\left(\vec{L}_{2}\left(\Omega, \rho_{0}\right)\right)$; moreover, $\|S\|=1$ (see $[6])$.

2. Let the boundary $S$ of the region $\Omega$ belong to the class $C^{2}$. Consider a boundary-value problem

$$
\left.-\rho_{0}^{-1}\left(\mu \Delta \vec{u}+\left(\eta+3^{-1} \mu\right) \nabla \operatorname{div} \vec{u}\right)=\vec{w} \quad(\text { in } \Omega), \quad \vec{u}=\overrightarrow{0} \quad \text { (on } S\right) .
$$

For every field $\vec{w} \in \vec{L}_{2}\left(\Omega, \rho_{0}\right)$, the generalized solution of the problem, given by the formula $\vec{u}=A^{-1} \vec{w}$, exists and it is unique. The operator $A$ is selfadjoint and positive definite in $\vec{L}_{2}\left(\Omega, \rho_{0}\right)$. The operator $A^{-1}$ belongs to the class $\mathfrak{S}_{p}(p>3 / 2)$. In addition, $\mathcal{D}\left(A^{1 / 2}\right)=\left\{\vec{u} \in \vec{W}_{2}^{1}(\Omega) \mid \vec{u}=\overrightarrow{0}(\right.$ on $\left.S)\right\}$.

3. Let us define an operator $B \vec{u}:=a_{\infty} \rho_{0}^{-1 / 2} \operatorname{div}\left(\rho_{0} \vec{u}\right), \mathcal{D}(B):=\mathcal{D}\left(A^{1 / 2}\right)$. The adjoint operator $B^{*} \rho=-\nabla\left(a_{\infty} \rho_{0}^{-1 / 2} \rho\right), \mathcal{D}\left(B^{*}\right)=W_{2}^{1}(\Omega) \cap L_{2, \rho_{0}}(\Omega)$; moreover, $B^{*} B A^{-1} \in \mathcal{L}\left(\vec{L}_{2}\left(\Omega, \rho_{0}\right)\right)$. 
4. The operator $Q:=B A^{-1 / 2}$ is bounded: $Q \in \mathcal{L}\left(\vec{L}_{2}\left(\Omega, \rho_{0}\right), L_{2, \rho_{0}}(\Omega)\right)$. The operator $Q^{+}:=A^{-1 / 2} B^{*}$ admits extension to the bounded operator $Q^{*}: \overline{Q^{+}}=Q^{*}$, $Q^{+}=\left.Q^{*}\right|_{\mathcal{D}\left(B^{*}\right)}$.

5. The operators $M_{l} \rho:=\Pi \rho_{0} k_{l} \Pi \rho(l=\overline{1, m})$ are bounded selfadjoint and positive definite in $L_{2, \rho_{0}}(\Omega)$. In addition, $M_{l} \mathcal{D}\left(B^{*}\right) \subset \mathcal{D}\left(B^{*}\right)(l=\overline{1, m})$.

Using the operators introduced above, we can write problem (1) as a system of two equations with initial-value conditions in a Hilbert space $H_{0}:=\vec{L}_{2}\left(\Omega, \rho_{0}\right) \oplus$ $L_{2, \rho_{0}}(\Omega)$ :

$$
\left\{\begin{array}{l}
\frac{d \vec{u}}{d t}+2 \omega_{0} i S \vec{u}+A \vec{u}-B^{*} \rho+\sum_{l=1}^{m} \int_{0}^{t} e^{-b_{l}(t-s)} B^{*} M_{l} \rho(s) d s=\vec{f}(t), \\
\frac{d \rho}{d t}+B \vec{u}=0, \quad(\vec{u}(0) ; \rho(0))^{\tau}=\left(\vec{u}^{0} ; \rho^{0}\right)^{\tau} .
\end{array}\right.
$$

The upper index $\tau$ denotes the transposition.

Definition 1.1. A strong solution to problem (2) is sad to be a strong solution to initial-value problem (1). A function $\zeta(t):=(\vec{u}(t) ; \rho(t))^{\tau}$ is a strong solution to problem (2) if $\zeta(t) \in \mathcal{D}(A) \oplus \mathcal{D}\left(B^{*}\right)$ for all $t \in \mathbb{R}_{+},\left(A \vec{u}(t) ; B^{*} \rho(t)\right)^{\tau} \in$ $C\left(\mathbb{R}_{+}, H_{0}\right),(\vec{u}(t) ; \rho(t))^{\tau} \in C^{1}\left(\mathbb{R}_{+}, H_{0}\right)$, and $\zeta(t)$ satisfies $(2)$ for all $t \in \mathbb{R}_{+}:=$ $[0,+\infty)$.

\subsection{Reduction to the first-order differential equation. The theorem on the solvability}

Let us suppose that problem (2) has a strong solution $\vec{u}(t), \rho(t)$. From Lemma 1 , it follows that $\vec{u}(t)$ and $\rho(t)$ satisfy the following system:

$$
\left\{\begin{array}{l}
\frac{d \vec{u}}{d t}+A^{1 / 2}\left\{2 \omega_{0} i A^{-1 / 2} S \vec{u}+A^{1 / 2} \vec{u}-Q^{+} \rho+\sum_{l=1}^{m} Q^{*} \int_{0}^{t} e^{-b_{l}(t-s)} M_{l} \rho(s) d s\right\}=\vec{f}(t), \\
\frac{d \rho}{d t}+Q A^{1 / 2} \vec{u}=0, \quad(\vec{u}(0) ; \rho(0))^{\tau}=\left(\vec{u}^{0} ; \rho^{0}\right)^{\tau} .
\end{array}\right.
$$

From $\rho(t) \in C^{1}\left(\mathbb{R}_{+}, L_{2, \rho_{0}}(\Omega)\right)$ and $M_{l} \in \mathcal{L}\left(L_{2, \rho_{0}}(\Omega)\right)$, we conclude that

$$
\int_{0}^{t} e^{-b_{l}(t-s)} M_{l} \rho(s) d s=\frac{1}{b_{l}} M_{l} \rho-\int_{0}^{t} e^{-b_{l}(t-s)} \frac{1}{b_{l}} M_{l} \frac{d \rho(s)}{d s} d s-e^{-b_{l} t} \frac{1}{b_{l}} M_{l} \rho^{0} .
$$

Let us suppose that $\rho^{0} \in \mathcal{D}\left(B^{*}\right)$. From the above, it follows that $\vec{u}(t)$ and 
$\rho(t)$ satisfy the system

$$
\left\{\begin{array}{l}
\frac{d \vec{u}}{d t}+A^{1 / 2}\left\{2 \omega_{0} i A^{-1 / 2} S \vec{u}+A^{1 / 2} \vec{u}-Q^{*}\left[I-\sum_{l=1}^{m} \frac{1}{b_{l}} M_{l}\right] \rho\right. \\
\left.\quad-\sum_{l=1}^{m} Q^{*} \int_{0}^{t} e^{-b_{l}(t-s)} \frac{1}{b_{l}} M_{l} \frac{d \rho(s)}{d s} d s\right\}=\vec{f}(t)+\sum_{l=1}^{m} e^{-b_{l} t} \frac{1}{b_{l}} B^{*} M_{l} \rho^{0} \\
\frac{d \rho}{d t}+Q A^{1 / 2} \vec{u}=0, \quad(\vec{u}(0) ; \rho(0))^{\tau}=\left(\vec{u}^{0} ; \rho^{0}\right)^{\tau} .
\end{array}\right.
$$

In what follows, we suppose that the physical parameters satisfy the condition

$$
\varphi_{0}(x):=1-\sum_{l=1}^{m} \frac{\rho_{0}(z) k_{l}(x)}{b_{l}}>0 \quad \forall x \in \bar{\Omega} .
$$

The condition supposes that the times of relaxation $b_{l}^{-1}$ and the structural functions $k_{l}(x)(l=\overline{1, m})$ are sufficiently small.

Let the function $g(x)$ be continuous $(g(x) \in C(\Omega))$ and satisfy the conditions $0<g_{1} \leqslant g(x) \leqslant g_{2}$. Define $L_{2, g}(\Omega):=\left\{f \in L_{2}(\Omega) \mid\left(f, g^{1 / 2}\right)_{L_{2}(\Omega)}=0\right\}$.

We introduce the operators $T_{l} \rho:=\left(k_{l} \rho_{0}\right)^{1 / 2} \Pi \rho(l=\overline{1, m}), T \rho:=\varphi_{0}^{1 / 2} \Pi \rho$. Using (4), it is easy to check that

$$
\begin{array}{cl}
T_{l},\left(T_{l}^{*}\right)^{-1} \in \mathcal{L}\left(L_{2, \rho_{0}}(\Omega), L_{2, k_{l}^{-1}}(\Omega)\right), & T_{l}^{-1}, T_{l}^{*} \in \mathcal{L}\left(L_{2, k_{l}^{-1}}(\Omega), L_{2, \rho_{0}}(\Omega)\right), \\
T,\left(T^{*}\right)^{-1} \in \mathcal{L}\left(L_{2, \rho_{0}}(\Omega), L_{2, \varphi_{0}^{-1} \rho_{0}}(\Omega)\right), & T^{-1}, T^{*} \in \mathcal{L}\left(L_{2, \varphi_{0}^{-1} \rho_{0}}(\Omega), L_{2, \rho_{0}}(\Omega)\right), \\
M_{l}=T_{l}^{*} T_{l}(l=\overline{1, m}), \quad I-\sum_{l=1}^{m} \frac{1}{b_{l}} M_{l}=T^{*} T .
\end{array}
$$

Let us define the following objects connected with the function $\rho(t)$ :

$$
T \rho(t)=: r(t), \quad \int_{0}^{t} e^{-b_{l}(t-s)} \frac{1}{\sqrt{b_{l}}} T_{l} \frac{d \rho(s)}{d s} d s=: r_{l}(t), \quad(l=\overline{1, m}) .
$$

The functions $r(t)$ and $r_{l}(t)(l=\overline{1, m})$ are continuously differentiable. From (3), it follows that these functions satisfy the system

$$
\left\{\begin{array}{cc}
\frac{d \vec{u}}{d t}+A^{1 / 2}\left\{2 \omega_{0} i A^{-1 / 2} S \vec{u}+A^{1 / 2} \vec{u}-Q^{*} T^{*} r-\sum_{l=1}^{m} \frac{1}{\sqrt{b_{l}}} Q^{*} T_{l}^{*} r_{l}\right\}=\vec{f}_{0}(t), \\
\frac{d r}{d t}+T Q A^{1 / 2} \vec{u}=0, \quad \frac{d r_{l}}{d t}+\frac{1}{\sqrt{b_{l}}} T_{l} Q A^{1 / 2} \vec{u}+b_{l} r_{l}=0 \quad(l=\overline{1, m}) \\
\overrightarrow{f_{0}}(t):=\vec{f}(t)+\sum_{l=1}^{m} e^{-b_{l} t} \frac{1}{b_{l}} B^{*} M_{l} \rho^{0} .
\end{array}\right.
$$


We will interpret this system (including initial data) as a Cauchy problem for a first-order differential equation in some Hilbert space. More precisely, we write system (6) as a first-order differential equation in a Hilbert space $\mathcal{H}:=H \oplus \mathcal{H}_{0}$ $\left(H:=\vec{L}_{2}\left(\Omega, \rho_{0}\right), \mathcal{H}_{0}:=L_{2, \varphi_{0}^{-1} \rho_{0}}(\Omega) \oplus_{l=1}^{m} L_{2, k_{l}^{-1}}(\Omega)\right)$ :

$$
\frac{d \xi}{d t}+\mathcal{A} \xi=\mathcal{F}(t), \quad \xi(0)=\xi^{0}
$$

where $\xi:=(\vec{u} ; w)^{\tau}, w:=\left(r ; r_{1} ; \ldots ; r_{m}\right)^{\tau}, \xi^{0}:=\left(\vec{u}^{0} ; w^{0}\right)^{\tau}, w^{0}:=\left(T \rho^{0} ; 0 ; \ldots ; 0\right)^{\tau}$, $\mathcal{F}(t):=\left(\overrightarrow{f_{0}}(t) ; 0 ; \ldots ; 0\right)^{\tau}$. The operator $\mathcal{A}$ satisfies the following formulae:

$$
\begin{gathered}
\mathcal{A}=\operatorname{diag}\left(A^{1 / 2}, \mathcal{I}_{0}\right)\left(\begin{array}{cc}
I & \mathcal{Q}^{*} \\
-\mathcal{Q} & \mathcal{G}
\end{array}\right) \operatorname{diag}\left(A^{1 / 2}, \mathcal{I}_{0}\right)+\left(\begin{array}{cc}
2 \omega_{0} i S & 0 \\
0 & 0
\end{array}\right), \\
\mathcal{A}=\left(\begin{array}{cc}
I & 0 \\
-\mathcal{Q} A^{-1 / 2} & \mathcal{I}_{0}
\end{array}\right) \operatorname{diag}\left(A, \mathcal{G}+\mathcal{Q} \mathcal{Q}^{*}\right)\left(\begin{array}{cc}
I & A^{-1 / 2} \mathcal{Q}^{*} \\
0 & \mathcal{I}_{0}
\end{array}\right)+\left(\begin{array}{cc}
2 \omega_{0} i S & 0 \\
0 & 0
\end{array}\right), \\
\mathcal{D}(\mathcal{A})=\left\{\xi=(\vec{u} ; w)^{\tau} \in \mathcal{H} \mid \vec{u}+A^{-1 / 2} \mathcal{Q}^{*} w \in \mathcal{D}(A)\right\},
\end{gathered}
$$

where $I$ and $\mathcal{I}_{0}$ are the identity operators in $H$ and $\mathcal{H}_{0}$, respectively,

$$
\mathcal{Q}:=\left(-T Q, \frac{-1}{\sqrt{b_{1}}} T_{1} Q, \ldots, \frac{-1}{\sqrt{b_{m}}} T_{m} Q\right)^{\tau}, \quad \mathcal{G}:=\operatorname{diag}\left(0, b_{1} I, \ldots, b_{m} I\right) .
$$

Definition 1.2. We say that a function $\xi(t)$ is a strong solution to Cauchy problem (7) if $\xi(t) \in \mathcal{D}(\mathcal{A})$ for all $t \in \mathbb{R}_{+}, \mathcal{A} \xi(t) \in C\left(\mathbb{R}_{+} ; \mathcal{H}\right), \xi(t) \in C^{1}\left(\mathbb{R}_{+} ; \mathcal{H}\right)$, and $\xi(t)$ satisfies $(7)$ for all $t \in \mathbb{R}_{+}$.

From the above, we make a conclusion that if $\rho^{0} \in \mathcal{D}\left(B^{*}\right)$ and Cauchy problem (2) has a strong solution $\vec{u}(t), \rho(t)$, then the function $\xi(t)$ is a strong solution to Cauchy problem (7).

Let us recall that the numerical range of the operator $\mathcal{A}$ is the set

$$
\mathcal{W}(\mathcal{A}):=\left\{(\mathcal{A} \xi, \xi)_{\mathcal{H}} \mid \xi \in \mathcal{D}(\mathcal{A}),\|\xi\|_{\mathcal{H}}=1\right\} \subset \mathbb{C} .
$$

Lemma 2. The operator $\mathcal{A}$ is maximal sectorial. Moreover,

$$
\begin{gathered}
\mathcal{W}(\mathcal{A}) \subset\left\{\lambda \in \mathbb{C}|| \operatorname{Im} \lambda \mid \leqslant\left\|\mathcal{Q}^{*}\right\|^{2}\left(2 \omega_{0}\right)^{-1} \operatorname{Re} \lambda+2 \omega_{0}, \quad 0 \leqslant \operatorname{Re} \lambda \leqslant 4 \omega_{0}^{2}\left\|\mathcal{Q}^{*}\right\|^{-2}\right\} \\
\cup\left\{\lambda \in \mathbb{C}|| \operatorname{Im} \lambda \mid \leqslant 2\left\|\mathcal{Q}^{*}\right\|(\operatorname{Re} \lambda)^{1 / 2}, \quad \operatorname{Re} \lambda \geqslant 4 \omega_{0}^{2}\left\|\mathcal{Q}^{*}\right\|^{-2}\right\} .
\end{gathered}
$$

P r o o f. First of all, it should be noted that $\mathcal{D}(A) \oplus \mathcal{D}^{*} \subset \mathcal{D}(\mathcal{A})$, where $\mathcal{D}^{*}:=$ $\left(T^{*}\right)^{-1} \mathcal{D}\left(B^{*}\right) \oplus_{l=1}^{m}\left(T_{l}^{*}\right)^{-1} \mathcal{D}\left(B^{*}\right)$, and thus the operator $\mathcal{A}$ is densely defined. Then the Schur-Frobenius factorization (9) of the operator $\mathcal{A}$ implies that the operator $\mathcal{A}$ is closed on $\mathcal{D}(\mathcal{A})$. 
Let us prove that the operator $\mathcal{A}$ is sectorial. Let $\xi=(\vec{u} ; w)^{\tau} \in \mathcal{D}(\mathcal{A})$. Then $\vec{u} \in \mathcal{D}\left(A^{1 / 2}\right)$. The symmetric factorization (8) of the operator $\mathcal{A}$ and Lemma 1 imply

$$
\begin{aligned}
& \operatorname{Re}(\mathcal{A} \xi, \xi)_{\mathcal{H}}=\operatorname{Re}\left(\left(\begin{array}{cc}
I & \mathcal{Q}^{*} \\
-\mathcal{Q} & \mathcal{G}
\end{array}\right)\left(\begin{array}{c}
A^{1 / 2} \vec{u} \\
w
\end{array}\right),\left(\begin{array}{c}
A^{1 / 2} \vec{u} \\
w
\end{array}\right)\right)_{\mathcal{H}}=\left\|A^{1 / 2} \vec{u}\right\|_{H}^{2}+\left\|\mathcal{G}^{1 / 2} w\right\|_{\mathcal{H}_{0}}^{2} \\
& \left|\operatorname{Im}(\mathcal{A} \xi, \xi)_{\mathcal{H}}\right|=\left|\operatorname{Im}\left[\left(\mathcal{Q}^{*} w, A^{1 / 2} \vec{u}\right)_{H}-\left(\mathcal{Q} A^{1 / 2} \vec{u}, w\right)_{\mathcal{H}_{0}}+2 \omega_{0} i(S \vec{u}, \vec{u})_{H}\right]\right| \\
& \quad=\left|2 \operatorname{Im}\left(\mathcal{Q}^{*} w, A^{1 / 2} \vec{u}\right)_{H}+2 \omega_{0} i(S \vec{u}, \vec{u})_{H}\right| \leqslant 2\left\|A^{1 / 2} \vec{u}\right\|_{H}\left\|\mathcal{Q}^{*} w\right\|_{H}+2 \omega_{0}\|\vec{u}\|_{H}^{2} .
\end{aligned}
$$

Hence, for any given $\alpha>0$,

$$
\begin{aligned}
& \operatorname{Re}(\mathcal{A} \xi, \xi)_{\mathcal{H}}-\alpha\left|\operatorname{Im}(\mathcal{A} \xi, \xi)_{\mathcal{H}}\right| \geqslant\left(\left\|A^{1 / 2} \vec{u}\right\|_{H}-\alpha\left\|\mathcal{Q}^{*} w\right\|_{H}\right)^{2} \\
& \quad-\alpha^{2}\left\|\mathcal{Q}^{*} w\right\|_{H}^{2}+\left\|\mathcal{G}^{1 / 2} w\right\|_{\mathcal{H}_{0}}^{2}-2 \omega_{0} \alpha\|\vec{u}\|_{H}^{2} \geqslant-\max \left\{\alpha^{2}\left\|\mathcal{Q}^{*}\right\|^{2}, 2 \omega_{0} \alpha\right\}\|\xi\|_{\mathcal{H}}^{2}
\end{aligned}
$$

Consequently,

$$
\operatorname{Re}([\mathcal{A}+\gamma(\alpha)] \xi, \xi)_{\mathcal{H}}-\alpha\left|\operatorname{Im}([\mathcal{A}+\gamma(\alpha)] \xi, \xi)_{\mathcal{H}}\right| \geqslant 0
$$

where $\gamma(\alpha):=\max \left\{\alpha^{2}\left\|\mathcal{Q}^{*}\right\|^{2}, 2 \omega_{0} \alpha\right\}$. Thus,

$$
\left|\operatorname{Im}([\mathcal{A}+\gamma(\alpha)] \xi, \xi)_{\mathcal{H}}\right| \leqslant \alpha^{-1} \operatorname{Re}([\mathcal{A}+\gamma(\alpha)] \xi, \xi)_{\mathcal{H}} \quad \forall \xi \in \mathcal{D}(\mathcal{A}), \quad \alpha>0 .
$$

It follows that $\mathcal{W}(\mathcal{A}) \subset\left\{\lambda \in \mathbb{C}|| \arg (\lambda+\gamma(\alpha)) \mid \leqslant \operatorname{arctg} \alpha^{-1}\right\}$ for all $\alpha>0$. In other words, the operator $\mathcal{A}$ is sectorial.

The formula from the lemma can be obtained by constructing an envelope curve for the corresponding family of lines.

To prove that the operator $\mathcal{A}$ is maximal, it suffices to show that the operator $\mathcal{A}-\lambda$ is continuously invertible for some $\lambda<0$. Let $\xi_{1}:=\left(\vec{u}_{1} ; w_{1}\right)^{\tau} \in \mathcal{D}(\mathcal{A})$, $\xi_{2}:=\left(\vec{u}_{2} ; w_{2}\right)^{\tau} \in \mathcal{H}$. From (8), it follows that the equation $(\mathcal{A}-\lambda) \xi_{1}=\xi_{2}$ can be written in the following form:

$$
\operatorname{diag}\left(A^{1 / 2}, \mathcal{I}_{0}\right)\left(\begin{array}{cc}
I+2 \omega_{0} i S_{A}-\lambda A^{-1} & \mathcal{Q}^{*} \\
-\mathcal{Q} & \mathcal{G}-\lambda
\end{array}\right) \operatorname{diag}\left(A^{1 / 2}, \mathcal{I}_{0}\right)\left(\begin{array}{c}
\vec{u}_{1} \\
w_{1}
\end{array}\right)=\left(\begin{array}{c}
\vec{u}_{2} \\
w_{2}
\end{array}\right)
$$

where $S_{A}:=A^{-1 / 2} S A^{-1 / 2}$. It can be easily seen that if the middle operator-block is continuously invertible, then the operator $\mathcal{A}-\lambda$ is continuously invertible.

Define $L(\lambda):=I-\lambda A^{-1}+2 \omega_{0} i S_{A}+\mathcal{Q}^{*}(\mathcal{G}-\lambda)^{-1} \mathcal{Q}$. It can be checked that $\operatorname{Re} L(\lambda) \gg 0, \operatorname{Re}(L(\lambda))^{*} \gg 0$ for all $\lambda<0$. Consequently, $L(\lambda)^{-1} \in \mathcal{L}(H)$, and direct calculations show that

$$
\begin{aligned}
& \left(\begin{array}{cc}
I-\lambda A^{-1}+2 \omega_{0} i S_{A} & \mathcal{Q}^{*} \\
-\mathcal{Q} & \mathcal{G}-\lambda
\end{array}\right)^{-1} \\
& \quad=\left(\begin{array}{cc}
L^{-1} & -L^{-1} \mathcal{Q}^{*}(\mathcal{G}-\lambda)^{-1} \\
(\mathcal{G}-\lambda)^{-1} \mathcal{Q} L^{-1} & (\mathcal{G}-\lambda)^{-1}-(\mathcal{G}-\lambda)^{-1} \mathcal{Q} L^{-1} \mathcal{Q}^{*}(\mathcal{G}-\lambda)^{-1}
\end{array}\right) \in \mathcal{L}(\mathcal{H})
\end{aligned}
$$


Using Lemma 2, we can prove the following theorem.

Theorem 1. Let the field $\vec{f}(t, x)$ satisfy the Hölder condition $\forall \tau \in \mathbb{R}_{+}$ $\exists K=K(\tau)>0, k(\tau) \in(0,1]:\|\vec{f}(t)-\vec{f}(s)\|_{\vec{L}_{2}\left(\Omega, \rho_{0}\right)} \leq K|t-s|^{k}$ for all $0 \leq s$, $t \leq \tau$. Then for any $\vec{u}^{0} \in \mathcal{D}(A)$ and $\rho^{0} \in \mathcal{D}\left(B^{*}\right)$ there exists a unique strong solution to initial-boundary value problem (1).

P r o o f. If $\vec{u}^{0} \in \mathcal{D}(A), \rho^{0} \in \mathcal{D}\left(B^{*}\right)$, then $\xi^{0} \in \mathcal{D}(\mathcal{A})$, where $\xi^{0}:=\left(\vec{u}^{0} ; w^{0}\right)^{\tau}$, $w^{0}:=\left(T \rho^{0} ; 0 ; \ldots ; 0\right)^{\tau}$. Indeed, from (10) and Lemma 1 it follows that

$$
\begin{aligned}
\vec{u}^{0}+A^{-1 / 2} \mathcal{Q}^{*} & w^{0}=\vec{u}^{0}-A^{-1 / 2} Q^{*} T^{*} T \rho^{0}=\vec{u}^{0}-\left.A^{-1 / 2} Q^{*}\right|_{\mathcal{D}\left(B^{*}\right)} T^{*} T \rho^{0} \\
= & \vec{u}^{0}-A^{-1 / 2} Q^{+} T^{*} T \rho^{0}=\vec{u}^{0}-A^{-1} B^{*}\left[I-\sum_{l=1}^{m} \frac{1}{b_{l}} M_{l}\right] \rho^{0} \in \mathcal{D}(A) .
\end{aligned}
$$

The assumption imposed on the field $\vec{f}(t)$ implies that the field $\vec{f}_{0}(t)$ satisfies the Hölder condition $\left(\overrightarrow{f_{0}}(t)=\vec{f}(t)+\sum_{l=1}^{m} e^{-b_{l} t} b_{l}^{-1} B^{*} M_{l} \rho^{0}\right.$, see (6)). It follows that the function $\mathcal{F}(t)$ defined in (7) also satisfies the Hölder condition.

By [7, Theorem 1.24, p. 492], the operator $-\mathcal{A}$ generates a strongly continuous semigroup of bounded operators. This semigroup is also holomorphic in the open sector which contains the positive real semiaxis. By [7, Theorem 1.27, p. 493], Cauchy problem (7) has a unique strong solution $\xi(t)$ (see Definition 1.2). From this fact one can deduce the rest of the proof.

\section{Essential Spectrum of a Rotating Viscous Relaxing Fluid}

In this section, we formulate a spectral problem associated with the problem on the spectrum of a rotating viscous relaxing fluid. The theorem on the essential and discrete spectrum is proven (Theorem 2).

\subsection{The basic spectral problems}

We consider the spectral problem to the evolution problem (7). Assuming $\mathcal{F}(t) \equiv 0$, a dependence on time for the unknown function is in the form $\xi(t)=\exp (-\lambda t) \xi$, where $\lambda$ is a spectral parameter and $\xi$ is an amplitude element, we have

$$
\mathcal{A} \xi=\lambda \xi, \quad \xi \in \mathcal{D}(\mathcal{A}) \subset \mathcal{H} .
$$

We associate problem (11) with the problem on the spectrum of a rotating viscous relaxing fluid.

Let $\xi=(\vec{u} ; w)^{\tau} \in \mathcal{D}(\mathcal{A})$. Let us change the sought element in problem (11), $\operatorname{diag}\left(A^{1 / 2}, \mathcal{I}_{0}\right) \xi=\zeta=:(\vec{v} ; w)^{\tau}$, using factorization (8). We have the following spectral problem $\left(S_{A}:=A^{-1 / 2} S A^{-1 / 2}\right)$ :

$$
\mathcal{A}(\lambda) \zeta:=\left(\begin{array}{cc}
I-\lambda A^{-1}+2 \omega_{0} i S_{A} & \mathcal{Q}^{*} \\
-\mathcal{Q} & \mathcal{G}-\lambda
\end{array}\right)\left(\begin{array}{c}
\vec{v} \\
w
\end{array}\right)=0, \quad \zeta \in \mathcal{H}=H \oplus \mathcal{H}_{0} .
$$


Let $\lambda \notin\left\{0, b_{1}, \ldots, b_{m}\right\}=\sigma(\mathcal{G})$. From (12), it follows that

$$
L(\lambda) \vec{v}:=\left[I-\lambda A^{-1}+2 \omega_{0} i S_{A}+\mathcal{Q}^{*}(\mathcal{G}-\lambda)^{-1} \mathcal{Q}\right] \vec{v}=0, \quad \vec{v} \in H .
$$

It is clear that the spectrum of the operator $\mathcal{A}$ coincides with the spectrum of the operator pencil $L(\lambda)$ for $\lambda \notin \sigma(\mathcal{G})$ (the spectrums of problem (12) and (13)).

To study the essential spectrum of the operator $\mathcal{A}$, we will need some auxiliary facts from the theory of elliptic boundary-value problems. In what follows we suppose that the boundary $S=\partial \Omega$ of the region $\Omega$ belongs to the class $C^{\infty}$.

\subsection{The local coordinate system and elliptic boundary-value prob- lems}

We are going to recall some definitions and introduce the auxiliary results from the theory of elliptic boundary-value problems (see [8-11] ). Let us consider a system of partial differential equations of order two or less,

$$
\mathcal{L}(x, D) u(x)=f(x), \quad x \in \Omega,
$$

where $x=\left(x_{1} ; x_{2} ; x_{3}\right) \in \Omega, D:=\left(D_{1} ; D_{2} ; D_{3}\right):=\left(-i \frac{\partial}{\partial x_{1}} ;-i \frac{\partial}{\partial x_{2}} ;-i \frac{\partial}{\partial x_{3}}\right), u(x):=$ $\left(u_{1}(x) ; \ldots ; u_{n}(x)\right)^{\tau}, f(x):=\left(f_{1}(x) ; \ldots ; f_{n}(x)\right)^{\tau}$. Denote by $\mathcal{L}(x, \xi), \xi:=\left(\xi_{1} ; \xi_{2} ; \xi_{3}\right)^{\tau}$ the polynomial matrix which can be obtained from (14) by substituting the symbol $D$ for $\xi$. In what follows we suppose system (14) defines nondegenerate Douglis-Nirenberg system (see [8, p. 375] and [9]).

Definition 2.3. (see [8]) The operator $\mathcal{L}(x, D)$ is sad to be elliptic in a closed region $\bar{\Omega}$ if $\pi \operatorname{det} \mathcal{L}(x, \xi) \neq 0$ for all $x \in \bar{\Omega}$ and $\xi \in \mathbb{R}^{3} \backslash\{0\}$. The symbol $\pi$ denotes the principal homogeneous part of the polynomial.

It is known that $\pi \operatorname{det} \mathcal{L}(x, \xi)=\operatorname{det} \pi \mathcal{L}(x, \xi)$, where $\pi \mathcal{L}$ is the principal part of the matrix $\mathcal{L}$ (see [8, p. 377] for obtaining the principal part of the DouglisNirenberg system).

Let us take an arbitrary point $z_{0} \in S$ and introduce a local coordinate system in its neighborhood (see $[10,11]$ ). Let the boundary $S$ be given locally by the infinitely differentiable functions $z_{i}=z_{i}\left(y_{1}, y_{2}\right), i=1,2,3$ in the variables $y_{1}$, $y_{2}$. The variables are chosen such that the coordinate lines $y_{i}=$ const are the curvature lines. We can write $z=z\left(y^{\prime}\right)$, where $y^{\prime}:=\left(y_{1} ; y_{2}\right)$. Denote by $N\left(y^{\prime}\right)$ the inner unit vector perpendicular to the boundary $S$. In the neighborhood of $S$, introduce the coordinates $y_{1}, y_{2}, y_{3}$, where $y_{3}$ is the distance from the point $x$ to $S$. Then $x=z\left(y^{\prime}\right)+y_{3} N\left(y^{\prime}\right)$. We enumerate $y_{1}, y_{2}$ so that the direction of the vector product $\partial z / \partial y_{1} \times \partial z / \partial y_{2}$ coincides with the inner unit normal $N\left(y^{\prime}\right)$. Let $E_{i}\left(y^{\prime}\right)(i=1,2)$ be the coefficients of the first quadratic form of the surface $S$. Then $\partial z / \partial y_{i} \cdot \partial z / \partial y_{j}=E_{i}\left(y^{\prime}\right) \delta_{i j}$, where $\delta_{i j}$ is the Kronecker delta. 
Let $\mathcal{B}(x, D)$ be a matrix of dimension $3 \times n$. The coefficients of this matrix are the linear differential operators of order one or less. Let us consider a system of boundary conditions

$$
\mathcal{B}(x, D) u(x)=g(x), \quad x \in S .
$$

In the constructed local coordinate system, the following formulae hold for the principal parts of the operators connected with boundary-value problem (14), (15):

$$
\pi \mathcal{L}\left(y,-i \frac{\partial}{\partial y}\right)=\pi \mathcal{L}\left(y,-i \frac{\partial}{\partial y^{\prime}},-i \frac{\partial}{\partial y_{3}}\right), \quad \pi \mathcal{B}\left(y,-i \frac{\partial}{\partial y}\right)=\pi \mathcal{B}\left(y,-i \frac{\partial}{\partial y^{\prime}},-i \frac{\partial}{\partial y_{3}}\right) .
$$

Definition 2.4. (see [8, p. 380], [10, p. 12]) Boundary-value problem (14), (15) is said to be elliptic if Definition 2.3 holds and the Shapiro-Lopatinsky condition

$$
\operatorname{rang} \int_{\gamma_{+}} \pi \mathcal{B}\left(\left(y^{\prime}, 0\right), \xi^{\prime}, \xi_{3}\right)\left[\pi \mathcal{L}\left(\left(y^{\prime}, 0\right), \xi^{\prime}, \xi_{3}\right)\right]^{-1}\left(I_{n}, \xi_{3} I_{n}\right) d \xi_{3}=3
$$

also holds for all $y^{\prime}$ from the domain of a local coordinate system for all $\xi^{\prime} \in \mathbb{R}^{2} \backslash\{0\}$ and $\xi_{3} \in \mathbb{R}$. Here $I_{n}$ denotes the unit matrix in $\mathbb{R}^{n},\left(I_{n}, \xi_{3} I_{n}\right)$ denotes the matrix of dimension $n \times 2 n, \gamma_{+}$denotes the rectifiable contour situated in the upper $\xi_{3}$ half-plane. This contour encloses in the positive direction all the $\xi_{3}$-roots of the equation $\operatorname{det} \pi \mathcal{L}\left(\left(y^{\prime}, 0\right), \xi^{\prime}, \xi_{3}\right)=0$, situated in the upper half-plane.

To verify the Shapiro-Lopatinsky condition, we need the following lemmata and notations from [10].

Lemma 3. (see $[10$, p. 14]) In the constructed local coordinate system the operators $\partial / \partial x_{i}$ have the form

$$
\frac{\partial}{\partial x_{i}}=\sum_{j=1}^{2}\left(1-K_{j} y_{3}\right)^{-1} E_{j}^{-1}\left(y^{\prime}\right) \frac{\partial z_{i}}{\partial y_{j}} \frac{\partial}{\partial y_{j}}+N_{i} \frac{\partial}{\partial y_{3}},
$$

where $K_{j}(j=1,2)$ are the principal curvatures of the surface $S$.

Let us introduce the following notations:

$$
\beta:=\left(\beta_{1} ; \beta_{2} ; \beta_{3}\right)^{\tau}, \quad \beta_{l}:=\sum_{j=1}^{2} E_{j}^{-1}\left(y^{\prime}\right) \frac{\partial z_{l}}{\partial y_{j}} \xi_{j} \quad(l=1,2,3), \quad \alpha:=\beta+\xi_{3} N .
$$

Then $\beta^{\tau} N=0, N^{\tau} \beta=0, N^{\tau} N=1$. Define $\left|\xi^{\prime}\right|^{2}:=|\beta|^{2}$. Then we have $\left|\xi^{\prime}\right|^{2}:=$ $\beta^{\tau} \beta=E_{1}^{-1} \xi_{1}^{2}+E_{2}^{-1} \xi_{2}^{2}$. In the local coordinate system the symbol $|\xi|^{2}$ means $|\xi|^{2}:=\left|\xi^{\prime}\right|^{2}+\xi_{3}^{2}$. 
Lemma 4. (see [10, p. 15]) In the introduced local coordinate system at $y_{3}=0$ the following formulae hold for the principal symbols of various operators:

$$
\sigma_{0}\left(\frac{\partial}{\partial x_{l}}\right)=i \alpha_{l}, \sigma_{0}(\nabla)=i \alpha, \sigma_{0}(\operatorname{div})=i \alpha^{\tau}, \sigma_{0}(\Delta)=-|\xi|^{2}=-\left(\left|\xi^{\prime}\right|^{2}+\xi_{3}^{2}\right) .
$$

Lemma 5. (see $\left[10\right.$, p. 16]) Let $\gamma_{+}$denote the rectifiable contour situated in the upper $\xi_{3}$-half-plane. The contour encloses the point $\xi_{3}=i\left|\xi^{\prime}\right|$ in the positive direction. Then the following formulae hold:

$$
\begin{gathered}
\int_{\gamma_{+}} \frac{d \xi_{3}}{|\xi|^{2}}=\frac{2 \pi}{2\left|\xi^{\prime}\right|}, \int_{\gamma_{+}} \frac{\xi_{3} d \xi_{3}}{|\xi|^{2}}=\frac{2 \pi i}{2}, \quad \int_{\gamma_{+}} \frac{\xi_{3}^{2} d \xi_{3}}{|\xi|^{2}}=-\frac{2 \pi\left|\xi^{\prime}\right|}{2}, \quad \int_{\gamma_{+}} \frac{d \xi_{3}}{|\xi|^{4}}=\frac{2 \pi}{4\left|\xi^{\prime}\right|^{3}}, \\
\int_{\gamma_{+}} \frac{\xi_{3} d \xi_{3}}{|\xi|^{4}}=0, \quad \int_{\gamma_{+}} \frac{\xi_{3}^{2} d \xi_{3}}{|\xi|^{4}}=\frac{2 \pi}{4\left|\xi^{\prime}\right|}, \quad \int_{\gamma_{+}} \frac{\xi_{3}^{3} d \xi_{3}}{|\xi|^{4}}=\frac{2 \pi i}{2}, \quad|\xi|^{2}=\left|\xi^{\prime}\right|^{2}+\xi_{3}^{2} .
\end{gathered}
$$

\subsection{On the essential and discrete spectrum of the problem}

Let us recall that a linear closed operator is sad to be Fredholm if its deficiency and index are finite (see [7, p. 229]).

Definition 2.5. We call a set

$$
\sigma_{\text {ess }}(\mathcal{A}):=\{\lambda \in \mathbb{C} \mid \mathcal{A}-\lambda \text { is not a Fredholm operator }\}
$$

the essential spectrum of the operator $\mathcal{A}$ (of problem (11)).

One can check that problem (11) defines nondegenerate Douglis-Nirenberg system. From [12] it follows that $\mathcal{A}-\lambda$ is a Fredholm operator if and only if the corresponding boundary-value problem is elliptic. The boundary-value problem connected with problem (11) has the following form (the first equation in the system is multiplied by $\left.\rho_{0}\right)$ :

$$
\left\{\begin{array}{cc}
-\left(\mu \Delta \vec{u}+\left(\eta+3^{-1} \mu\right) \nabla \operatorname{div} \vec{u}\right)+\rho_{0} \nabla\left(a_{\infty} \rho_{0}^{-1 / 2} \varphi_{0}^{1 / 2} r\right) & \\
+\rho_{0} \sum_{l=1}^{m} \frac{1}{b_{l}^{1 / 2}} \nabla\left(a_{\infty} k_{l}^{1 / 2} r_{l}\right)-2 \omega_{0} \rho_{0}\left(\vec{u} \times e_{3}\right)-\lambda \rho_{0} \vec{u}=0 & \\
a_{\infty} \rho_{0}^{-1 / 2} \varphi_{0}^{1 / 2} \operatorname{div}\left(\rho_{0} \vec{u}\right)-\lambda r=0, & (l=\overline{1, m}) \quad(\text { in } \Omega), \\
a_{\infty} k_{l}^{1 / 2} b_{l}^{-1 / 2} \operatorname{div}\left(\rho_{0} \vec{u}\right)+\left(b_{l}-\lambda\right) r_{l}=0 & \vec{u}=\overrightarrow{0} \text { (on } S) .
\end{array}\right.
$$


Define $\mu^{\prime}:=\eta+3^{-1} \mu$ and extract the principal parts from system (17) and boundary condition (18):

$$
\left\{\begin{array}{l}
-\left(\mu \Delta \vec{u}+\mu^{\prime} \nabla \operatorname{div} \vec{u}\right)+a_{\infty} \rho_{0}^{1 / 2} \varphi_{0}^{1 / 2} \nabla r+\sum_{l=1}^{m} \frac{a_{\infty} \rho_{0} k_{l}^{1 / 2}}{b_{l}^{1 / 2}} \nabla r_{l}=0, \\
a_{\infty} \rho_{0}^{1 / 2} \varphi_{0}^{1 / 2} \operatorname{div} \vec{u}-\lambda r=0, \\
a_{\infty} \rho_{0} k_{l}^{1 / 2} b_{l}^{-1 / 2} \operatorname{div} \vec{u}+\left(b_{l}-\lambda\right) r_{l}=0 \quad(l=\overline{1, m}) \quad(\text { in } \Omega), \\
\vec{u}=\overrightarrow{0} \quad(\text { on } S) .
\end{array}\right.
$$

Denote by $\mathcal{L}_{\lambda}(x, D)$ the matrix differential operator of system (17). It is a matrix of dimension $(m+4) \times(m+4)$. Denote by $\mathcal{B}(x, D):=\left(I_{3}, 0_{3 \times m+1}\right)$ the matrix corresponding to boundary condition (18). It is a matrix of dimension $3 \times(m+4)$. In this case, the principal part $\pi \mathcal{L}_{\lambda}(x, D)$ of the operator $\mathcal{L}_{\lambda}(x, D)$ is defined by system $(19)$, and $\pi \mathcal{B}(x, D)=\mathcal{B}(x, D)$.

Hence the essential spectrum of our problem is the set of all $\lambda \in \mathbb{C}$ such that the ellipticity of boundary-value problem (17), (18) is broken.

Lemma 6. The differential operator $\mathcal{L}_{\lambda}(x, D)$ is elliptic in the closed region $\bar{\Omega} \subset \mathbb{R}^{3}$ for all $\lambda \notin \Lambda_{E}$, where

$$
\Lambda_{E}:=\left\{\lambda \in \mathbb{C} \mid \varphi_{\lambda}(x):=1-\sum_{l=1}^{m} \frac{\rho_{0}(z) k_{l}(x)}{b_{l}-\lambda}=\lambda \frac{\mu+\mu^{\prime}}{a_{\infty}^{2} \rho_{0}(z)}, x \in \bar{\Omega}\right\} .
$$

$\mathrm{P} \mathrm{r}$ o o f. Let us consider the principal symbol $\sigma_{0}\left(\mathcal{L}_{\lambda}(x, D)\right)=\pi \mathcal{L}_{\lambda}(x, \xi)$ $\left(\xi:=\left(\xi_{1} ; \xi_{2} ; \xi_{3}\right)^{\tau}\right)$ of system (17) which is defined by system (19):

$$
\pi \mathcal{L}_{\lambda}(x, \xi)=\left(\begin{array}{ccccc}
\mu|\xi|^{2} I_{3}+\mu^{\prime} \xi \xi^{\tau} & c_{0} i \xi & c_{1} i \xi & \ldots & c_{m} i \xi \\
c_{0} i \xi^{\tau} & -\lambda & 0 & \ldots & 0 \\
c_{1} i \xi^{\tau} & 0 & b_{1}-\lambda & 0_{1 \times m-2} & 0 \\
\ldots & \ldots & \ldots & \ldots & \ldots \\
c_{m} i \xi^{\tau} & 0 & 0 & 0_{1 \times m-2} & b_{m}-\lambda
\end{array}\right) .
$$

Here we define $c_{0}=c_{0}(x):=a_{\infty} \rho_{0}^{1 / 2}(z) \varphi_{0}^{1 / 2}(x), c_{l}=c_{l}(x):=a_{\infty} b_{l}^{-1 / 2} \rho_{0}(z) k_{l}^{1 / 2}(x)$ $(l=\overline{1, m}) .0_{1 \times m-2}$ denotes the trivial matrix of dimension $1 \times(m-2)$.

Define $\Gamma_{\xi}:=\left(|\xi|^{-1} \xi, a^{\perp}, b^{\perp}\right)$, where the column-vectors $a^{\perp}, b^{\perp}\left(\left|a^{\perp}\right|=\left|b^{\perp}\right|=1\right)$ are orthogonal to $\xi$ and to each other. One can easily check that

$$
\Gamma_{\xi}^{\tau} \Gamma_{\xi}=I_{3}, \Gamma_{\xi}^{\tau} \xi=(|\xi|, 0,0)^{\tau}=:|\xi| e_{1}, \Gamma_{\xi}^{\tau} \xi \xi^{\tau} \Gamma_{\xi}=\operatorname{diag}\left(|\xi|^{2}, 0,0\right)=:|\xi|^{2} P_{1} .
$$


Define $\mathcal{S}:=\operatorname{diag}(\Gamma_{\xi}, \underbrace{1, \ldots, 1}_{m+1 \text { times }})$. From $(22),(23)$, it follows that

$$
\begin{aligned}
& \pi \operatorname{det} \mathcal{L}(x, \xi)=\operatorname{det} \pi \mathcal{L}(x, \xi)=\operatorname{det} \mathcal{S}^{\tau} \pi \mathcal{L}(x, \xi) \mathcal{S} \\
&= \operatorname{det}\left(\begin{array}{ccccc}
|\xi|^{2}\left(\mu I_{3}+\mu^{\prime} P_{1}\right) & c_{0} i|\xi| e_{1} & c_{1} i|\xi| e_{1} & \ldots & c_{m} i|\xi| e_{1} \\
c_{0} i|\xi| e_{1}^{\tau} & -\lambda & 0 & \ldots & 0 \\
c_{1} i|\xi| e_{1}^{\tau} & 0 & b_{1}-\lambda & 0_{1 \times m-2} & 0 \\
\ldots & \ldots & \ldots & \ldots & \ldots \\
c_{m} i|\xi| e_{1}^{\tau} & 0 & 0 & 0_{1 \times m-2} & b_{m}-\lambda
\end{array}\right) \\
&=|\xi|^{6} \mu^{2}\left(\left(c_{0}^{2}-\lambda\left(\mu+\mu^{\prime}\right)\right) \prod_{l=1}^{m}\left(b_{l}-\lambda\right)-\lambda \sum_{l=1}^{m} c_{l}^{2} \prod_{k=1, k \neq l}^{m}\left(b_{k}-\lambda\right)\right) .
\end{aligned}
$$

One can easily see that if $\lambda=b_{l}(l=\overline{1, m})$, then $\pi \operatorname{det} \mathcal{L}_{\lambda}(x, \xi) \neq 0$ for all $x \in \bar{\Omega}$ and $\xi \in \mathbb{R}^{3} \backslash\{0\}$. Using (4) and (22), we transform (24):

$$
\pi \operatorname{det} \mathcal{L}_{\lambda}(x, \xi)=a_{\infty}^{2} \rho_{0}(z) \mu^{2}|\xi|^{6} \prod_{l=1}^{m}\left(b_{l}-\lambda\right)\left(1-\lambda \frac{\mu+\mu^{\prime}}{a_{\infty}^{2} \rho_{0}(z)}-\sum_{l=1}^{m} \frac{\rho_{0}(z) k_{l}(x)}{b_{l}-\lambda}\right) .
$$

If $\lambda \notin \Lambda_{E}$, then from (25) we conclude that $\pi \operatorname{det} \mathcal{L}_{\lambda}(x, \xi) \neq 0$ for all $x \in \bar{\Omega}$ and $\xi \in \mathbb{R}^{3} \backslash\{0\}$.

To prove the next two lemmata, we need the following fact. Let $A_{k l} \in \mathcal{L}(H)$ $(k, l=1,2), A_{22}^{-1} \in \mathcal{L}(H), D_{1}:=A_{11}-A_{12} A_{22}^{-1} A_{21}$. If $D_{1}^{-1} \in \mathcal{L}(H)$, then there exists an inverse matrix

$$
\begin{aligned}
\left(\begin{array}{ll}
A_{11} & A_{12} \\
A_{21} & A_{22}
\end{array}\right)^{-1} & =\left[\left(\begin{array}{cc}
I & A_{12} A_{22}^{-1} \\
0 & I
\end{array}\right)\left(\begin{array}{cc}
D_{1} & 0 \\
0 & A_{22}
\end{array}\right)\left(\begin{array}{cc}
I & 0 \\
A_{22}^{-1} A_{21} & I
\end{array}\right)\right]^{-1} \\
& =\left(\begin{array}{c}
D_{1}^{-1} \\
-A_{22}^{-1} A_{21} D_{1}^{-1}
\end{array} \quad A_{22}^{-1}\left[\begin{array}{c}
-D_{1}^{-1} A_{12} A_{22}^{-1} \\
\left.A_{21} D_{1}^{-1} A_{12}\right] A_{22}^{-1}
\end{array}\right) .\right.
\end{aligned}
$$

We also use the following formulae:

$$
\begin{aligned}
\left(\begin{array}{cc}
a|\xi|^{2} I_{3}+b \alpha \alpha^{\tau} & i c \alpha \\
i c \alpha^{\tau} & 0
\end{array}\right)^{-1} & =\left(\begin{array}{cc}
a^{-1}|\xi|^{-4}\left(|\xi|^{2} I_{3}-\alpha \alpha^{\tau}\right) & -c^{-1}|\xi|^{-2} i \alpha \\
-c^{-1}|\xi|^{-2} i \alpha^{\tau} & c^{-2}(a+b)
\end{array}\right) \\
\left(a|\xi|^{2} I_{3}+b \alpha \alpha^{\tau}\right)^{-1} & =\frac{(a+b)|\xi|^{2} I_{3}-b \alpha \alpha^{\tau}}{a(a+b)|\xi|^{4}}
\end{aligned}
$$

which are true for all $a \neq 0, c \neq 0, a+b \neq 0$ and $\xi \in \mathbb{R}^{3} \backslash\{0\}$.

Lemma 7. Let $\lambda=0, \lambda=b_{l}(l=\overline{1, m})$. Then problem (17), (18) is elliptic. 
P r o o f. By Definition 2.4 and Lemma 6 , we have to verify the ShapiroLopatinsky condition for problem (17), (18).

Let us take an arbitrary point $z_{0} \in S$ and introduce the local coordinate system in its neighborhood (see Sec. 2.2). We write the matrix differential operator of system (17) in the local coordinate system and take its principal part. This principal part is the matrix differential operator of system (19) written in the local coordinate system and obtained from (22) by substituting $\xi$ by $\alpha$. The symbol $\operatorname{det} \pi \mathcal{L}_{\lambda}\left(\left(y^{\prime}, 0\right), \xi^{\prime}, \xi_{3}\right)$ can be obtained from (25) (if $\lambda=b_{l}(l=\overline{1, m})$ then from (24) ) by substituting $|\xi|^{2}$ by $\left|\xi^{\prime}\right|^{2}+\xi_{3}^{2}$. If $\lambda=0, \lambda=b_{l}(l=\overline{1, m})$, then the equation $\operatorname{det} \pi \mathcal{L}_{\lambda}\left(\left(y^{\prime}, 0\right), \xi^{\prime}, \xi_{3}\right)=0$ has the triple $\xi_{3}$-roots $\xi_{3}= \pm i\left|\xi^{\prime}\right|$.

Let $\lambda=0$. Using (26) (with $A_{22}=\operatorname{diag}\left(b_{1}, \ldots, b_{m}\right)$ ) and (27) (with $a:=\mu$ ), we find the matrix which is an inverse matrix of $\pi \mathcal{L}_{0}\left(\left(y^{\prime}, 0\right), \xi^{\prime}, \xi_{3}\right)$. Taking into account $\pi \mathcal{B}\left(\left(y^{\prime}, 0\right), \xi^{\prime}, \xi_{3}\right)=\left(I_{3}, 0_{3 \times m+1}\right)$, we calculate the rank of the following matrix:

$$
\begin{aligned}
\int_{\gamma_{+}} \pi \mathcal{B}\left(\left(y^{\prime}, 0\right), \xi^{\prime}, \xi_{3}\right)\left[\pi \mathcal{L}_{0}\left(\left(y^{\prime}, 0\right), \xi^{\prime}, \xi_{3}\right)\right]^{-1}\left(I_{m+4}, \xi_{3} I_{m+4}\right) d \xi_{3} \\
=\int_{\gamma_{+}}\left(\frac{|\xi|^{2} I_{3}-\alpha \alpha^{\tau}}{\mu|\xi|^{4}}, \mathcal{M}_{3 \times m+1}^{(1)}\right)\left(I_{m+4}, \xi_{3} I_{m+4}\right) d \xi_{3},
\end{aligned}
$$

where $\mathcal{M}_{3 \times m+1}^{(1)}$ is some matrix of dimension $3 \times(m+1)$.

From (16) and Lemma 5, it follows that

$$
M:=\int_{\gamma_{+}} \frac{|\xi|^{2} I_{3}-\alpha \alpha^{\tau}}{\mu|\xi|^{4}} d \xi_{3}=\frac{\pi}{2 \mu\left|\xi^{\prime}\right|^{3}}\left(2\left|\xi^{\prime}\right|^{2} I_{3}-\beta \beta^{\tau}-\left|\xi^{\prime}\right|^{2} N N^{\tau}\right) .
$$

Define $\Gamma_{\alpha}:=\left(\left|\xi^{\prime}\right|^{-1} \beta, a^{\perp}, N\right)$, where the column-vector $a^{\perp}\left(\left|a^{\perp}\right|=1\right)$ is orthogonal to $\beta$ and to $N$. One can easily check that

$$
\begin{aligned}
& \Gamma_{\alpha}^{\tau} \Gamma_{\alpha}=I_{3}, \quad \Gamma_{\alpha}^{\tau} \beta=\left(\left|\xi^{\prime}\right|, 0,0\right)^{\tau}=:\left|\xi^{\prime}\right| e_{1}, \quad \Gamma_{\alpha}^{\tau} N=(0,0,1)^{\tau}=: e_{3}, \\
& \Gamma_{\alpha}^{\tau} \beta \beta^{\tau} \Gamma_{\alpha}=\operatorname{diag}\left(\left|\xi^{\prime}\right|^{2}, 0,0\right)=:\left|\xi^{\prime}\right|^{2} P_{1}, \quad \Gamma_{\alpha}^{\tau} N N^{\tau} \Gamma_{\alpha}=\operatorname{diag}(0,0,1)=: P_{3}
\end{aligned}
$$

Using (31), (32), we transform (30) and see that if $\xi^{\prime} \neq 0$, then

$$
\operatorname{det} M=\operatorname{det} \Gamma_{\alpha}^{\tau} M \Gamma_{\alpha}=\frac{\pi^{3}}{8 \mu^{3}\left|\xi^{\prime}\right|^{9}} \operatorname{det}\left(2\left|\xi^{\prime}\right|^{2} I_{3}-\left|\xi^{\prime}\right|^{2} P_{1}-\left|\xi^{\prime}\right|^{2} P_{3}\right)=\frac{\pi^{3}}{4 \mu^{3}\left|\xi^{\prime}\right|^{3}} \neq 0 .
$$

It follows that if $\xi^{\prime} \neq 0$, then the rank of matrix (29) is equal to 3. By Definition 2.4, problem (17), (18) is elliptic.

In the same way, the ellipticity of problem (17), (18) can be verified for the case $\lambda=b_{l}(l=\overline{1, m})$. 
Lemma 8. Problem (17), (18) is elliptic if $\lambda \notin \Lambda_{E} \cup \Lambda_{L}$, where

$$
\Lambda_{L}:=\left\{\lambda \in \mathbb{C} \mid \varphi_{\lambda}(x):=1-\sum_{l=1}^{m} \frac{\rho_{0}(z) k_{l}(x)}{b_{l}-\lambda}=\lambda \frac{2 \mu+\mu^{\prime}}{a_{\infty}^{2} \rho_{0}(z)}, x \in S\right\} .
$$

The set $\Lambda_{E}$ is defined in Lemma 6 (see (21)).

P r o o f. First of all, we assume that $\lambda \notin \Lambda_{E}$. If $\lambda \in \Lambda_{E}$, then the operator $\mathcal{L}_{\lambda}(x, D)$ is not elliptic (see Lemma 6). From this it follows that problem (17), (18) is not elliptic (see Definition 2.4). We also assume that $\lambda \neq 0, \lambda \neq b_{l}(l=\overline{1, m})$. If $\lambda=0$ or $\lambda=b_{l}(l=\overline{1, m})$, then problem (17), (18) is elliptic (see Lemma 7).

Let us take an arbitrary point $z_{0} \in S$ and introduce the local coordinate system in its neighborhood (see Sec. 2.2). Using (26) (with $A_{22}=\operatorname{diag}\left(-\lambda, b_{1}-\right.$ $\left.\lambda, \ldots, b_{m}-\lambda\right)$ ) and (28) (with $a:=\mu, b:=\mu^{\prime}-a_{\infty}^{2} \rho_{0} \lambda^{-1} \varphi_{\lambda}$ ), we find the matrix which is an inverse matrix of $\pi \mathcal{L}_{\lambda}\left(\left(y^{\prime}, 0\right), \xi^{\prime}, \xi_{3}\right)$. We calculate the rank of the matrix

$$
\begin{aligned}
\int_{\gamma_{+}} \pi \mathcal{B}\left(\left(y^{\prime}, 0\right), \xi^{\prime}, \xi_{3}\right)\left[\pi \mathcal{L}_{\lambda}\left(\left(y^{\prime}, 0\right), \xi^{\prime}, \xi_{3}\right)\right]^{-1}\left(I_{m+4}, \xi_{3} I_{m+4}\right) d \xi_{3} \\
=\int_{\gamma_{+}}\left(\frac{(\mu+b)|\xi|^{2} I_{3}-b \alpha \alpha^{\tau}}{\mu(\mu+b)|\xi|^{4}}, \mathcal{M}_{3 \times m+1}^{(2)}\right)\left(I_{m+4}, \xi_{3} I_{m+4}\right) d \xi_{3}
\end{aligned}
$$

where $\mathcal{M}_{3 \times m+1}^{(2)}$ is some matrix of dimension $3 \times(m+1)$.

From (16) and Lemma 5, it follows that

$$
M:=\int_{\gamma_{+}} \frac{(\mu+b)|\xi|^{2} I_{3}-b \alpha \alpha^{\tau}}{\mu(\mu+b)|\xi|^{4}} d \xi_{3}=\frac{\pi}{2 \mu\left|\xi^{\prime}\right|^{3}}\left(2\left|\xi^{\prime}\right|^{2} I_{3}-\frac{b}{\mu+b} \beta \beta^{\tau}-\frac{b}{\mu+b}\left|\xi^{\prime}\right|^{2} N N^{\tau}\right) .
$$

Using (31), (32), we transform the matrix $M$. Taking into account $b=\mu^{\prime}-$ $a_{\infty}^{2} \rho_{0} \lambda^{-1} \varphi_{\lambda}$, we see that if $\lambda \notin \Lambda_{L}$ and $\xi^{\prime} \neq 0$, then

$$
\begin{aligned}
\operatorname{det} M & =\operatorname{det} \Gamma_{\alpha}^{\tau} M \Gamma_{\alpha}=\frac{\pi^{3}}{8 \mu^{3}\left|\xi^{\prime}\right|^{9}} \operatorname{det}\left(2\left|\xi^{\prime}\right|^{2} I_{3}-\frac{b}{\mu+b}\left(\left|\xi^{\prime}\right|^{2} P_{1}+\left|\xi^{\prime}\right|^{2} P_{3}\right)\right) \\
& =\frac{\pi^{3}\left(2 \mu+b\left(z_{0}\right)\right)^{2}}{4 \mu^{3}\left(\mu+b\left(z_{0}\right)\right)^{2}\left|\xi^{\prime}\right|^{3}}=\frac{\pi^{3}\left(2 \mu+\mu^{\prime}-a_{\infty}^{2} \rho_{0}\left(z_{0}\right) \lambda^{-1} \varphi_{\lambda}\left(z_{0}\right)\right)^{2}}{4 \mu^{3}\left(\mu+\mu^{\prime}-a_{\infty}^{2} \rho_{0}\left(z_{0}\right) \lambda^{-1} \varphi_{\lambda}\left(z_{0}\right)\right)^{2}\left|\xi^{\prime}\right|^{3}} \neq 0 .
\end{aligned}
$$

It follows that if $\xi^{\prime} \neq 0$, then the rank of matrix (34) is equal to 3. By Definition 2.4, problem (17), (18) is elliptic.

Theorem 2. $\sigma_{\text {ess }}(\mathcal{A})=\Lambda_{E} \cup \Lambda_{L}$. The set $\mathbb{C} \backslash \sigma_{\text {ess }}(\mathcal{A})$ consists of regular points and isolated eigenvalues of finite multiplicity of the operator $\mathcal{A}$. 
P r o o f. Definition 2.5 and Lemmata 7, 8 imply the formula for the essential spectrum of the operator $\mathcal{A}$. Lemma 2 implies that the operator $\mathcal{A}$ is maximal sectorial (and accretive). Consequently, if $\lambda<0$, then the operator $\mathcal{A}-\lambda$ is continuously invertible and its deficiency and index are equal to zero. It is obvious that the set $\mathbb{C} \backslash \sigma_{\text {ess }}(\mathcal{A})$ is connected. From this and [7, Theorem 5.17, p. 235] (The stability theorem for the index) it follows that the set $\mathbb{C} \backslash \sigma_{\text {ess }}(\mathcal{A})$ consists of regular points and isolated eigenvalues of finite multiplicity of the operator $\mathcal{A}$.

$\mathrm{R}$ e $\mathrm{m}$ a r k 2.1. If we let $\omega_{0}=0$ and $g=0$ in (1), then $\rho_{0}(z)=$ const and $k_{l}(x)$ $(l=\overline{1, m})$ are some positive structural constants satisfying (4). In this case, the essential spectrum $\sigma_{\text {ess }}(\mathcal{A})$ consists of the finite number of positive real numbers.

\section{Spectrum Localization}

In this section, we prove that $\lambda=0$ is not an eigenvalue of the operator $\mathcal{A}$ (of problem (11)). The spectrum of the operator $\mathcal{A}$ is situated in the open right half-plane and is localized near the real axis (Theorem 3).

In the case where the system is not rotating $\left(\omega_{0}=0\right)$, there can be only the finite number of nonreal eigenvalues of the operator $\mathcal{A}$. These complex eigenvalues are situated symmetrically with respect to the real axis. The rest of the spectrum is localized on the positive real semiaxis. The nonreal eigenvalues are absent if the viscosity of the fluid is sufficiently large (Theorem 4).

\subsection{Spectrum localization for the general case}

Some statements given below contain the conditions on the smallness of the norm $\left\|A^{-1 / 2}\right\|$. The norm $\left\|A^{-1 / 2}\right\|$ is decreasing when the maximum of the function $\mu \rho_{0}^{-1}(z)$ in the region $\Omega$ is increasing. Thus, the term "the norm $\left\|A^{-1 / 2}\right\|$ is sufficiently small" is equivalent to the term "the viscosity of the fluid is sufficiently large" (for $\omega_{0}=0$ and $g=0$, "the kinematic coefficient of viscosity $\nu=\mu \rho_{0}^{-1}$ of the fluid is sufficiently large").

Lemma 9. The point $\lambda=0$ is not an eigenvalue of the operator $\mathcal{A}$. If $\left\|A^{-1 / 2}\right\|<b_{q}^{-1 / 2}$, then $\lambda=b_{q}$ is not an eigenvalue of the operator $\mathcal{A}$. Otherwise, the point $\lambda=b_{q}$ can be an eigenvalue of finite multiplicity of the operator $\mathcal{A}$.

P r o o f. Theorem 2 implies that the points $\lambda=0, \lambda=b_{l}(l=\overline{1, m})$ can only be isolated eigenvalues of finite multiplicity of the operator $\mathcal{A}$.

Let $\lambda=0$ be an eigenvalue of the operator $\mathcal{A}$. Then there exists $0 \neq \zeta=$ $(\vec{v} ; w)^{\tau} \in \mathcal{H}$ such that $\mathcal{A}(0) \zeta=0$ (see (12)). From this it follows that $\vec{v}+$ $2 \omega_{0} i S_{A} \vec{v}+\mathcal{Q}^{*} w=0,-\mathcal{Q} \vec{v}+\mathcal{G} w=0$. Let us multiply these equations by $\vec{v}$ and $w$, respectively. It follows that $\|\vec{v}\|^{2}+\left\|\mathcal{G}^{1 / 2} w\right\|^{2}=0$. Consequently, $\vec{v}=0$, 
$r_{1}=\cdots=r_{m}=0, Q^{*} T^{*} r=0$. From this and $\operatorname{Ker} Q^{*} T^{*}=\{0\}$ it follows that $\zeta=0$. Hence, $\lambda=0$ is not an eigenvalue of the operator $\mathcal{A}$.

Let us write the resolvent equation $(\mathcal{A}-\lambda) \xi=\xi_{0}$ as the following system:

$$
\left\{\begin{aligned}
A^{1 / 2}\left[A^{1 / 2} \vec{u}-Q^{*} T^{*} r-\sum_{l=1}^{m} \frac{1}{\sqrt{b_{l}}} Q^{*} T_{l}^{*} r_{l}\right]+2 \omega_{0} i S \vec{u} & -\lambda \vec{u}=\vec{u}_{0}, \\
T Q A^{1 / 2} \vec{u}-\lambda r & =r_{0}, \\
\frac{l}{\sqrt{b_{l}}} T_{l} Q A^{1 / 2} \vec{u}+\left(b_{l}-\lambda\right) r_{l} & =r_{l 0}, \quad l=\overline{1, m}
\end{aligned}\right.
$$

Let $\lambda=b_{q}(q=\overline{1, m})$. Set $l=q\left(\lambda=b_{q}, \xi_{0}=0\right)$ in the third line in (35). It follows that $Q A^{1 / 2} \vec{u}=0$. Let us multiply the first equation in (35) by $\vec{u}$. From the last line in (35) it follows that $\left\|A^{1 / 2} \vec{u}\right\|_{H}^{2}+2 \omega_{0} i(S \vec{u}, \vec{u})_{H}-b_{q}\|\vec{u}\|_{H}^{2}=0$ or $\left\|A^{1 / 2} \vec{u}\right\|_{H}^{2}-b_{q}\|\vec{u}\|_{H}^{2}=0$. If $\left\|A^{-1 / 2}\right\|<b_{q}^{-1 / 2}$, then $\vec{u}=0$. This implies $\xi=0$. Hence $\lambda=b_{q}$ is not an eigenvalue of the operator $\mathcal{A}$.

Let us study the localization of the spectrum of the operator $\mathcal{A}$.

Theorem 3. 1. For any given $\varepsilon>0$, the spectrum of the operator $\mathcal{A}$, except the finite number of eigenvalues, is localized in $\left\{\lambda \in \mathbb{C}|\operatorname{Re} \lambda>0,| \operatorname{Im} \lambda \mid \leqslant 2 \omega_{0}+\varepsilon\right\}$.

2. The spectrum of the operator $\mathcal{A}$ includes the set of eigenvalues $\left\{\lambda_{n}^{(+\infty)}\right\}_{n=1}^{\infty}$, and these eigenvalues satisfy the following formula:

$$
\begin{gathered}
\lambda_{n}^{(+\infty)}=\lambda_{n}(A)(1+o(1)) \quad(n \rightarrow+\infty), \\
\lambda_{n}(A)=\left[\frac{2 \mu^{-3 / 2}+\left(\mu+\mu^{\prime}\right)^{-3 / 2}}{6 \pi^{2}} \int_{\Omega} \rho_{0}^{3 / 2}(z) d \Omega\right]^{-2 / 3} n^{2 / 3}(1+o(1)) \quad(n \rightarrow+\infty) .
\end{gathered}
$$

$\mathrm{P}$ r o o f. Let us prove the second statement. Fix $\varepsilon>0$. The operator $S_{A}$ is compact, $\operatorname{Ker} A^{-1}=\{0\}$. Hence, $\left\|\left(I-\lambda A^{-1}\right)^{-1} S_{A}\right\| \rightarrow 0$ as $\lambda \rightarrow \infty$, $\lambda \in \mathbb{C} \backslash\{|\arg \lambda|<\varepsilon\}$ (see [13, Lemma 3.1, p. 15]). It follows that there exists $R=R(\varepsilon)$ such that the pencil $L(\lambda)$ (see (13)) is continuously invertible in $\mathbb{C} \backslash(\{|\arg \lambda|<\varepsilon\} \cup\{|\lambda|<R\})$. The power asymptotics of eigenvalues of the operator $A$ and the theorem by A.S. Marcus and V.I. Matsaev (see [14]) imply the existence of the set of eigenvalues $\left\{\lambda_{n}^{(+\infty)}\right\}_{n=1}^{\infty}$ and the corresponding formula. The power asymptotics of eigenvalues of the operator $A$ follows from $[15, \mathrm{p} .10]$.

Let us prove the first statement. It is sufficient to show that the set of all eigenvalues is included in $\left\{\lambda \in \mathbb{C}|\operatorname{Re} \lambda>0,| \operatorname{Im} \lambda \mid \leqslant 2 \omega_{0}+\varepsilon\right\}$. Let $\lambda$ and $\vec{v}$ be an eigenvalue and an eigenvector of the pencil $L(\lambda)$, i.e., $L(\lambda) \vec{v}=0$. Let us multiply 
this equation by $\vec{v}$. It follows that

$$
\begin{gathered}
1-\lambda p+2 \omega_{0} i s-\frac{1}{\lambda}\left(q-\sum_{l=1}^{m} \frac{q_{l}}{b_{l}-\lambda}\right)=0 \\
p:=\frac{\left\|A^{-1 / 2} \vec{v}\right\|^{2}}{\|\vec{v}\|^{2}}, \quad s:=\frac{\left(S_{A} \vec{v}, \vec{v}\right)}{\|\vec{v}\|^{2}}, \quad q:=\frac{\|Q \vec{v}\|^{2}}{\|\vec{v}\|^{2}}, \quad q_{l}:=\frac{\left\|T_{l} Q \vec{v}\right\|^{2}}{\|\vec{v}\|^{2}} \quad(l=\overline{1, m}) .
\end{gathered}
$$

Let us extract the real and the imaginary parts from (36). We have

$$
\begin{gathered}
1-p \operatorname{Re} \lambda-q \frac{\operatorname{Re} \lambda}{|\lambda|^{2}}+\sum_{l=1}^{m} \frac{q_{l}}{b_{l}}\left(\frac{\operatorname{Re} \lambda}{|\lambda|^{2}}+\frac{b_{l}-\operatorname{Re} \lambda}{\left|b_{l}-\lambda\right|^{2}}\right)=0, \\
-p \operatorname{Im} \lambda+2 \omega_{0} s+q \frac{\operatorname{Im} \lambda}{|\lambda|^{2}}+\sum_{l=1}^{m} \frac{q_{l}}{b_{l}}\left(\frac{-\operatorname{Im} \lambda}{|\lambda|^{2}}+\frac{\operatorname{Im} \lambda}{\left|b_{l}-\lambda\right|^{2}}\right)=0 .
\end{gathered}
$$

Inequalities $p>0, q-\sum_{l=1}^{m} q_{l} b_{l}^{-1}=\|T Q \vec{v}\|^{2}\|\vec{v}\|^{-2} \geqslant 0$, and (37) imply $\operatorname{Re} \lambda>0$. From (38) and (37), we obtain

$$
\begin{aligned}
|\operatorname{Im} \lambda|=\left|2 \omega_{0} s\right|\left|p-\left[\frac{q}{|\lambda|^{2}}-\sum_{l=1}^{m} \frac{q_{l}}{b_{l}}\left(\frac{1}{|\lambda|^{2}}-\frac{1}{\left|b_{l}-\lambda\right|^{2}}\right)\right]\right|^{-1} & =\frac{\left|2 \omega_{0} s \operatorname{Re} \lambda\right|}{\left|2 p \operatorname{Re} \lambda-1-\sum_{l=1}^{m} \frac{q_{l}}{\left|b_{l}-\lambda\right|^{2}}\right|}=\frac{2 \omega_{0}|s|}{p} \cdot \frac{p \operatorname{Re} \lambda}{\left|2 p \operatorname{Re} \lambda-1-\sum_{l=1}^{m} \frac{q_{l}}{\left|b_{l}-\lambda\right|^{2}}\right|} .
\end{aligned}
$$

Lemma 1 implies $|s| p^{-1}=\left(S A^{-1 / 2} \vec{v}, A^{-1 / 2} \vec{v}\right)_{H}\left\|A^{-1 / 2} \vec{v}\right\|_{H}^{-2} \leqslant 1$. From (37), it follows that $p \operatorname{Re} \lambda \rightarrow 1$ as $\lambda \rightarrow \infty$. If the absolute magnitude of the eigenvalue $\lambda$ is sufficiently large, then (39) implies $|\operatorname{Im} \lambda| \leqslant 2 \omega_{0}+\varepsilon$.

\subsection{Spectrum localization for the case $\omega_{0}=0$}

Let us study the localization of the spectrum of the operator $\mathcal{A}$ in the case where the system is not rotating, i.e., $\omega_{0}=0$.

Theorem 4. Let us suppose that $\omega_{0}=0$.

1. There can be only the finite number of nonreal eigenvalues of the operator $\mathcal{A}$. These complex eigenvalues are situated symmetrically with respect to the real axis. The rest of the spectrum is localized on the real axis.

2. If $\lambda_{0}$ is a nonreal eigenvalue of the operator $\mathcal{A}$, then

$$
\begin{gathered}
{\left[2\left\|A^{-1 / 2}\right\|^{2}\right]^{-1}<\operatorname{Re} \lambda_{0}<b_{m}+\|Q\|^{2}+\|Q\|\left(b_{m}+\|Q\|^{2}\right)^{1 / 2}} \\
\left|\lambda_{0}\right|^{2}<\left(b_{m}+2\|Q\|^{2}+2\|Q\|\left(b_{m}+\|Q\|^{2}\right)^{1 / 2}\right)\left(2 b_{m}+\|Q\|^{2}\right) .
\end{gathered}
$$


The spectrum of the operator $\mathcal{A}$ is real if the following condition holds:

$$
2\left\|A^{-1 / 2}\right\|^{2} \leqslant\left(b_{m}+\|Q\|^{2}+\|Q\|\left(b_{m}+\|Q\|^{2}\right)^{1 / 2}\right)^{-1} .
$$

$\mathrm{P} \mathrm{r} \mathrm{o} \mathrm{o} \mathrm{f.} \mathrm{The} \mathrm{proof} \mathrm{of} \mathrm{the} \mathrm{first} \mathrm{statement} \mathrm{is} \mathrm{based} \mathrm{on} \mathrm{the} \mathrm{theory} \mathrm{of} \mathrm{spaces}$ with indefinite metrics and is mostly the verification of the fact that the operator $\mathcal{A}$ belongs to Helton's class $(\mathcal{A} \in(H))$. In the next paper we intend to study the root elements of the operator $\mathcal{A}$ and prove that $\mathcal{A} \in(H)$.

Let $\omega_{0}=0$, and $\lambda_{0}$ be a nonreal eigenvalue of the operator $\mathcal{A}$. Then there exists $\vec{v}=\vec{v}_{0}$ such that $\lambda_{0}$ is a root of equation (36). Let us write (36) in the form

$$
\begin{aligned}
0= & \left(\lambda-\lambda^{2} p-q\right) \prod_{l=1}^{m}\left(b_{l}-\lambda\right)+\sum_{l=1}^{m} q_{l} \prod_{k \neq l}^{m}\left(b_{k}-\lambda\right)=-p(-1)^{m} \lambda^{m+2} \\
& +(-1)^{m} \lambda^{m+1}\left[1+p \sum_{l=1}^{m} b_{l}\right]-(-1)^{m} \lambda^{m}\left[q+\sum_{l=1}^{m} b_{l}+p \sum_{i<j} b_{i} b_{j}\right]+\ldots
\end{aligned}
$$

Equation (36) has $m$ real roots, which we denote by $\lambda_{l}\left(\lambda_{l} \in\left(b_{l-1}, b_{l}\right), l=\overline{1, m}\right.$, $\left.b_{0}:=0\right)$, and two more roots, $\lambda_{0}$ and $\overline{\lambda_{0}}$. Define $\xi_{0}:=\operatorname{Re} \lambda_{0}, \eta_{0}:=\operatorname{Im} \lambda_{0}$. Then

$$
\begin{array}{r}
0=-p \prod_{l=1}^{m}\left(\lambda_{l}-\lambda\right)\left(\left(\lambda-\xi_{0}\right)^{2}+\eta_{0}^{2}\right)=-p(-1)^{m} \lambda^{m+2}+(-1)^{m} \lambda^{m+1} p\left[2 \xi_{0}+\sum_{l=1}^{m} \lambda_{l}\right] \\
-(-1)^{m} \lambda^{m} p\left[\left(\xi_{0}^{2}+\eta_{0}^{2}\right)+2 \xi_{0} \sum_{l=1}^{m} \lambda_{l}+\sum_{i<j} \lambda_{i} \lambda_{j}\right]+\ldots
\end{array}
$$

Equating the coefficients of $\lambda^{m+1}$ and $\lambda^{m}$ in (41), (42), we have

$$
\begin{gathered}
2 \xi_{0}+\sum_{l=1}^{m} \lambda_{l}=\frac{1}{p}+\sum_{l=1}^{m} b_{l} \\
\left(\xi_{0}^{2}+\eta_{0}^{2}\right)+2 \xi_{0} \sum_{l=1}^{m} \lambda_{l}+\sum_{i<j} \lambda_{i} \lambda_{j}=\frac{q}{p}+\frac{1}{p} \sum_{l=1}^{m} b_{l}+\sum_{i<j} b_{i} b_{j} .
\end{gathered}
$$

From (43), it follows that $2 \operatorname{Re} \lambda_{0}=2 \xi_{0}=p^{-1}+\sum_{l=1}^{m}\left(b_{l}-\lambda_{l}\right)>p^{-1} \geqslant$ $\left\|A^{-1 / 2}\right\|^{-2}$. Hence, $\operatorname{Re} \lambda_{0} \geqslant\left[2\left\|A^{-1 / 2}\right\|^{2}\right]^{-1}$.

Further, we follows the ideas of [16, p. 378]. Define $\delta:=2^{-1} \sum_{l=1}^{m}\left(b_{l}-\lambda_{l}\right)$, $\omega:=(2 p)^{-1}$. Then $\xi_{0}=\omega+\delta$ (see (43)). Extract $\sum_{l=1}^{m} \lambda_{l}$ from (43) and substitute it into (44). It follows that

$$
\eta_{0}^{2}+2 \delta \sum_{l=1}^{m} \lambda_{l}-\sum_{i<j}\left(b_{i} b_{j}-\lambda_{i} \lambda_{j}\right)=-\omega^{2}+2 \omega(\delta+q)-\delta^{2} .
$$


From $\lambda_{l} \in\left(b_{l-1}, b_{l}\right), l=\overline{1, m}\left(b_{0}:=0\right)$, one can obtain the following inequality (see $[16$, p. 380, Formula (5.24)]):

$$
\sum_{i<j}\left(b_{i} b_{j}-\lambda_{i} \lambda_{j}\right)<\sum_{j=1}^{m}\left(b_{j}-\lambda_{j}\right)\left(\sum_{i=1}^{m} \lambda_{i}\right)=2 \delta \sum_{l=1}^{m} \lambda_{l} .
$$

From (46), it follows that the right-hand part in (45) is positive. Consequently, $\omega<\delta+q+\left(2 \delta q+q^{2}\right)^{1 / 2}$. Therefore, $\operatorname{Re} \lambda_{0}=\xi_{0}<2 \delta+q+\left(2 \delta q+q^{2}\right)^{1 / 2} \leqslant$ $b_{m}+\|Q\|^{2}+\|Q\|\left[b_{m}+\|Q\|^{2}\right]^{1 / 2}$. Hence, $\operatorname{Re} \lambda_{0}<b_{m}+\|Q\|^{2}+\|Q\|\left(b_{m}+\|Q\|^{2}\right)^{1 / 2}$.

From the above we obtain (40).

Extract $\left(\xi_{0}^{2}+\eta_{0}^{2}\right)=\left|\lambda_{0}\right|^{2}$ from (44) and substitute it into (43). Using (46), we get $\left|\lambda_{0}\right|^{2}<2 \omega(q+4 \delta)$. From this we obtain the inequality for $\left|\lambda_{0}\right|^{2}$.

$\mathrm{R}$ e $\mathrm{m}$ a $\mathrm{r} \mathrm{k}$ 3.2. Let us note here that condition (40) can be simplified. More precisely, one can obtain condition $\left\|A^{-1 / 2}\right\| \leqslant\left(3 b_{m}+4\|Q\|^{2}\right)^{-1 / 2}$ instead of (40).

The author thanks Professor N.D. Kopachevsky for attention to this work, Professor A.N. Kozhevnikov, and the anonymous referee for useful suggestions.

\section{References}

[1] P.K. Pal and V.N. Maslennikova, Spectral Properties of Operators in a Problem on Normal Oscillations of a Compressible Fluid Filling Rotating Vessel. - Acad. Sci. USSR 281 (1985), No. 3, 529-534. (Russian)

[2] G. Geymonat and E. Sanchez-Palencia, On the Vanishing Viscosity Limit for Acoustic Phenomena in a Bounded Region. - Archiv for Rat. Mech. and Anal. 75 (1981), $257-268$.

[3] V.V. Vlasov and D.A. Medvedev, Functional-Differentional Equations in Sobolev Spaces and Connected Questions on Spectral Theory. - Modern Math. Fundamental Concepts 30 (2008), 3-173. (Russian)

[4] V.V. Vlasov, N.A. Rautian and A.S. Shamaev, Spectral Analysis and Well-Posed Solvability of Abstract Integro-Differential Equations Arising in Thermal Physics and Acoustics. - Modern Math. Fundamental Concepts 39 (2011), 36-65. (Russian)

[5] D. Zakora, A Symmetric Model of Viscous Relaxing Fluid. An Evolution Problem. - J. Math. Phys., Anal., Geom. 8 (2012), No. 2, 190-206.

[6] N.D. Kopachevsky, S.G. Krein and Ngo Zui Kan, Operator Methods in Linear Hydrodynamics. Evolution and Spectral Problems. Nauka, Moscow, 1989. (Russian)

[7] T. Kato, Perturbation Theory for Linear Operators. Springer-Verlag, BerlinHeidelberg-New York, 1980.

[8] L.R. Volevich, Solvability of Boundary-Value Problems for General Elliptic Systems. - Math. Sb. 68(110) (1965), No. 3, 373-416. (Russian) 
[9] V.A. Solonnikov, On General Boundary-Value Problems for A. Douglis-L. Nirenberg Elliptic Systems. II — Trudy Math. V.A. Steklov Ins. (1966), 233-297. (Russian)

[10] A.N. Kozhevnikov, Functional Methods of Mathematical Physics. Uchebnoe Posobie, MAI, Moscow, 1991. (Russian)

[11] A. Kozhevnikov and T. Skubachevskaya, Some Applications of Pseudo-Differential Operators to Elasticity. — Hokkaido Mathematical Journal 26 (1997), 297-322.

[12] G. Grubb and G. Geymonat, The Essential Spectrum of Elliptic Systems of Mixed Order. — Math. Ann. 227 (1977), 247-276.

[13] A.S. Marcus, Introduction to Spectral Theory of Polinomial Operator Pencils. Shtiinca, Kishenev, 1986. (Russian)

[14] A.S. Marcus and V.I. Matsaev, Theorem on Compare of Spectrums and Spectral Asymptotics for M.V. Keldysh's Pencil. — Math. Sb. 123(165) (1984), No. 3, 391-406. (Russian)

[15] M.Sh. Birman and M.Z. Solomjak, Asymptotics of Spectrum of Differential Equations. - Itogi Nauki i Tehniki VINITI. Seria Math. An. 14 (1977), 5-58. (Russian)

[16] N.D. Kopachevsky and S.G. Krein, Operator Approach to Linear Problems of Hydrodynamics. Vol. 2: Nonself-Adjoint Problems for Viscous Fluids. Birkhäuser Verlag, Basel-Boston-Berlin, 2003. 